\title{
Atherosclerosis Plaque Heterogeneity and Response to Therapy Detected by In Vivo Molecular Imaging of Matrix Metalloproteinase Activation
}

\author{
Mahmoud Razavian ${ }^{1,2}$, Sina Tavakoli ${ }^{1,2}$, Jiasheng Zhang ${ }^{1,2}$, Lei Nie ${ }^{1,2}$, Lawrence W. Dobrucki ${ }^{1}$, Albert J. Sinusas ${ }^{1}$, \\ Michael Azure ${ }^{3}$, Simon Robinson ${ }^{3}$, and Mehran M. Sadeghi ${ }^{1,2}$ \\ ${ }^{1}$ Cardiovascular Molecular Imaging Laboratory, Section of Cardiovascular Medicine, Yale University School of Medicine, New \\ Haven, Connecticut; ${ }^{2}$ VA Connecticut Healthcare System, West Haven, Connecticut; and ${ }^{3}$ Lantheus Medical Imaging, North Billerica, \\ Massachusetts
}

\begin{abstract}
Matrix metalloproteinases (MMPs) play a key role in the development of atherosclerosis and its complications. In vivo detection and quantification of MMP activation can help track the propensity to complications and response to therapy. We sought to establish an in vivo imaging approach for monitoring MMP activation in atherosclerotic mouse aorta and use it to assess the response to dietary modification. Method: Apolipoprotein-deficient mice were fed normal chow or a high-fat diet (HFD) for up to 3 mo or a HFD for 2 mo, followed by 1 mo on normal chow. Then they underwent micro-SPECT/CT, along with autoradiography and oil red $\mathrm{O}$ staining of tissues. Results: After 3 mo of HFD, there was considerable atherosclerosis in the aorta. In vivo micro-SPECT/CT using RP782 (an ${ }^{111} \mathrm{In}-$ labeled tracer targeting activated MMPs) showed a heterogeneous pattern of tracer uptake along the aorta. Heterogeneity of RP782 uptake was confirmed by autoradiography, and specificity was demonstrated using excess unlabeled precursor. Tracer uptake quantified by micro-SPECT significantly correlated with uptake quantified by autoradiography. Comparison of oil red $\mathrm{O}$ staining with autoradiography demonstrated areas of discordance between plaque presence and tracer uptake. HFD withdrawal led to significant reduction in RP782 uptake beyond the effect on plaque area. MMP expression and macrophage infiltration were similarly heterogeneous along the aorta and significantly reduced after withdrawal from the HFD. Finally, RP782 uptake significantly correlated with aortic macrophage content. Conclusion: Molecular imaging of MMP activation reveals the heterogeneity of atherosclerotic plaques and is a useful tool for tracking plaque biology and response to therapy.
\end{abstract}

Key Words: atherosclerosis; imaging; matrix metalloproteinases; nuclear medicine

J Nucl Med 2011; 52:1795-1802

DOI: 10.2967/jnumed.111.092379

\footnotetext{
Received Apr. 26, 2011; revision accepted Jul. 14, 2011.

For correspondence or reprints contact: Mehran M. Sadeghi, VA Connecticut Healthcare System, 950 Campbell Ave., 111B, West Haven, CT 06516.

E-mail: Mehran.sadeghi@yale.edu

Published online Oct. 3, 2011

COPYRIGHT @ 2011 by the Society of Nuclear Medicine, Inc.
}

A therosclerotic plaques often develop near branching segments of the artery where flow-mediated changes in shear stress are believed to contribute to early endothelial activation and injury that culminate in advanced atherosclerotic lesions. In addition to spatial heterogeneity, a key characteristic of atherosclerosis is the considerable heterogeneity in plaque structure and composition. High-risk plaques exhibit specific anatomic and biologic features, including the presence of a thin fibrous cap, large necrotic core, outward remodeling, and large number of inflammatory cells. The inflammatory cells promote a protease (including matrix metalloproteinase [MMP])-rich milieu that leads to plaque rupture and the resulting acute coronary syndromes $(1,2)$. Preventive and therapeutic measures (e.g., dietary intervention) aim at ameliorating these pathologic features to reduce the incidence of plaque rupture. However, despite several effective measures introduced in recent years, atherosclerosis remains a morbid disease. Recent advances in vessel wall imaging present an opportunity to detect in vivo structural and biologic features of atherosclerotic plaque that affect the clinical outcome $(3,4)$.

Murine models are valuable experimental tools for studies of atherosclerosis biology. Similar to human disease, murine atherosclerosis consists of heterogeneous lesions with a variety of cellular and biologic compositions. Molecular imaging of targets such as MMP activation, angiogenesis, and apoptosis (5-7) can potentially provide unique quantitative information on plaque biology, including determinants of morbid events and response to therapeutic interventions. However, the small size of the aorta has often limited the quantification of imaging targets in the mouse to ex vivo measurements or in vivo measurements without ex vivo validation (5,8-10). Here, we detect and quantify MMP activation associated with atherogenesis by micro-SPECT/CT in vivo, show its considerable heterogeneity along the aorta in high-fat-fed apolipoprotein E-deficient $\left(\mathrm{apoE}^{-/-}\right)$mice, and demonstrate an effect of dietary intervention on MMP activation in atherosclerosis that extends beyond the effect on plaque development. 


\section{MATERIALS AND METHODS}

\section{Reagents}

Reagents were obtained from Sigma, unless otherwise specified. RP782, an ${ }^{111}$ In-labeled tracer with specificity for activated MMPs, was provided by Lantheus Medical Imaging. The structure, binding characteristics, and biodistribution of MMP tracers in mice were previously reported $(5,11,12)$.

\section{Animal Model}

Six- to 8-wk-old female apoE $\mathrm{E}^{-1-}$ mice (Jackson Laboratory) were fed a high-cholesterol diet (1.25\% cholesterol; Harlan Teklad) ad libitum for $1 \mathrm{mo}(n=10), 2 \mathrm{mo}(n=8)$, or $3 \mathrm{mo}(n=10)$. In an additional group of animals, the high-fat diet (HFD) was replaced with normal chow after 2 mo $(n=10)$. Other animals were fed with normal chow for 3 mo $(n=3)$. Experiments were performed according to regulations of the Animal Care and Use Committees of the VA Connecticut Healthcare System and Yale University.

\section{Imaging}

Thirty-seven $( \pm 6.3) \mathrm{MBq}$ of RP782 $\left({ }^{111}\right.$ In-labeled tracer targeting MMP activation epitope) (11) were administered to animals through a right jugular vein catheter placed under anesthesia (isofluorane, $1 \%-3 \%$ ). Animals were imaged after $2 \mathrm{~h}$ on a highresolution small-animal imaging system (X-SPECT; Gamma Medica Ideas) with 1-mm medium-energy pinhole collimators (12). This system has a spatial resolution of approximately $2 \mathrm{~mm}$ for ${ }^{111} \mathrm{In}$. Anesthetized mice were placed in a fixed position on the animal bed. Three point sources (37-185 kBq) were placed in the field of view to verify the accuracy of image fusion. The following acquisition parameters were used for micro-SPECT: $360^{\circ}, 128$ projections, $30 \mathrm{~s} /$ projection $(\sim 80$-min image acquisition), 174- and $242-\mathrm{keV}$ photopeaks $\pm 10 \%$ window, and $82 \times 82$ matrix. After completion of micro-SPECT, animals were injected with a continuous infusion of iodinated CT contrast (iohexol, 350 $\mathrm{mg} / \mathrm{mL}$; rate, $100 \mu \mathrm{L} / \mathrm{min}$ ) over $2 \mathrm{~min}$, and CT was performed (energy, $75 \mathrm{kVp} / 280 \mu \mathrm{A}$; matrix, $512 \times 512$ ) to identify anatomic structures. To demonstrate tracer uptake specificity, 2 animals fed the HFD for 1 mo were injected with a 50-fold excess of unlabeled precursor 15 min before RP782 administration. The imaging protocol lasted approximately $1.5 \mathrm{~h}$, after which $(3.5 \mathrm{~h}$ after tracer administration) aortae were harvested for autoradiography.

Micro-SPECT images were reconstructed by iterative reconstruction using system software (X-Flex). CT projection images were reconstructed with Cobra EXIM software, which implements a conebeam reconstruction algorithm. Reconstructed micro-SPECT images were reoriented according to the CT anatomic images, fused, and exported in the Interfile format for further processing with AMIDE (a medical imaging data examiner) (isotropic voxel size, $0.314 \mathrm{~mm}$ ). For quantitative analysis of tracer uptake, 9 serial cylindric $(2 \times 2 \times 2$ $\mathrm{mm}$ ) regions of interest were drawn along the aorta starting at the level of the right brachiocephalic artery and ending proximal to the branching off of the renal arteries. A region of interest immediately anterior to the thoracic descending aorta was used to calculate the background activity. Data were expressed as background-corrected counts per voxel per megabecquerel (cpv/MBq) injected. Despite the presence of potentially confounding factors, for example, scatter and partial-volume effects, this approach yielded an excellent correlation with ex vivo measurements of tracer uptake. Multiplanar reconstruction was performed on successive images (thickness, $\sim 0.314 \mathrm{~mm}$ ) of the aorta and inferior vena cava (IVC) using EViewBox (Java Medical Imaging Software).

\section{Autoradiography}

Explanted aortae were exposed to high-sensitivity, x-radiographic X-OMAT Kodak Scientific Imaging Film (Eastman Kodak) for various times to optimize detection. The linearity of the signal was confirmed using a set of standards with known activity deposited on absorbent paper, which also served for tracer uptake quantification. Signal intensity was quantified on serial $2 \times 2 \mathrm{~mm}$ regions of interest drawn along the aorta starting at the level of the right brachiocephalic artery using Kodak 1D software (Kodak Scientific Imaging Systems). Data were background-corrected and expressed as percentage injected dose. The small size of aortic sections and their dehydration during autoradiography prevented accurate weight measurements.

\section{Oil Red O Staining}

Aortae were fixed in $4 \%$ formaldehyde. Staining with oil red O was performed according to a standard protocol. Briefly, after being rinsed with water, the aorta was exposed to isopropanol (60\%) for 2 min, followed by $1 \mathrm{~h}$ of staining with a solution of oil red $\mathrm{O}$ dissolved in isopropanol $(10 \mathrm{mg} / \mathrm{mL})$ and diluted to $60 \%$ in water. The excess stain was removed with isopropanol and water, and the aorta was photographed using a microscope equipped with a digital camera (Leica). The red-stained areas representing atherosclerotic lesions were measured using ImageJ software (National Institutes of Health), as previously described (13). The results were expressed as the ratio of total plaque area over total vessel area.

\section{Quantitative Reverse-Transcription Polymerase Chain Reaction (RT-PCR)}

Starting at the level of the right brachiocephalic artery, 4 consecutive 400- $\mu \mathrm{m}$ segments of the aorta, each at 4-mm intervals, were used for RNA isolation. Total RNA was isolated from each aortic section using an Absolutely RNA Nanoprep Kit (Stratagene) and reverse-transcribed using a QuantiTect Reverse Transcription Kit (Qiagen). Quantitative PCR was performed on this complementary DNA in triplicate using Taqman primers (Applied Biosystems) and an Applied Biosystems 7500 Real-Time PCR system following the manufacturer's instructions. The results were normalized to glyceraldehyde 3-phosphate dehydrogenase (GAPDH). The following primer sets were used: MMP-2 (Mm00439506_m1), MMP-3 (Mm00440295_m1), MMP-9 (Mm00442991_m1), MMP-12 (Mm00500554_m1), MMP-13 (Mm01168713_m1), CD68 (Mm00839636_g1), EMR1 (Mm00802529_m1), smooth muscle (SM) $\alpha$-actin (Mm01546133_m1), CD31 (Mm00476702_m1), and GAPDH (Mm99999915_g1).

\section{Statistical Analysis}

Statistical analysis was performed using GraphPad Prism (GraphPad Software). Data are presented as mean \pm SE. Differences between 2 groups were tested using a 2-tailed unpaired Student $t$ test. Multiple groups were compared using 1-way ANOVA with the Dunn multiple comparison test or 2-way ANOVA followed by Bonferroni post hoc analysis. Pearson correlation was used to test the association between 2 variables. Significance was set at the 0.05 level.

\section{RESULTS}

\section{MMP Activation Heterogeneity in Atherosclerotic Aorta}

To investigate MMP activation patterns in native atherosclerotic lesions, apoE $\mathrm{E}^{-/-}$mice were fed either an HFD or 
normal chow for 3 mo. In animals fed with normal chow, oil red $\mathrm{O}$ staining demonstrated the presence of a few fatty deposits along the aorta (Fig. 1). After 3 mo of HFD, there was extensive atherosclerosis along the aorta, with the most prominent lesions localized in the proximal aorta (Fig. 1). In these animals, micro-SPECT of MMP activation with ${ }^{111}$ In-labeled RP782 $(11,12)$, a radiotracer that specifically targets the MMP activation epitope, followed by CT angiography for anatomic localization demonstrated a heterogeneous MMP activation pattern along the aorta (Fig. 2; Supplemental Fig. 1 [supplemental materials are available online only at http://jnm.snmjournals.org]). Despite the larger size and blood volume of the IVC, little tracer uptake could be detected, which served as a control for imaging studies. The heterogeneous pattern of RP782 uptake was more readily visible after multiplanar reformation of the aorta based on CT angiography images (Fig. 2B). In vivo quantification of RP782 uptake showed significantly higher mean uptake of the tracer in the aorta than in the IVC $(0.36 \pm 0.05$ vs. $0.09 \pm 0.06 \mathrm{cpv} / \mathrm{MBq}, n=4, P=0.04)$, with the highest tracer uptake localized in the proximal aorta (Fig. 2C). The heterogeneous pattern of tracer uptake along the aorta was confirmed by ex vivo autoradiography (Figs. $3 \mathrm{~A}$ and $3 \mathrm{~B})$. There was a strong correlation between in vivo and ex vivo quantification of RP782 uptake along the aorta ( $r=0.68, P<0.001$, Fig. 3C). Oil red $\mathrm{O}$ staining of explanted aortae demonstrated that although there was in general a good match between the presence of atherosclerosis and RP782 uptake in the aorta, areas of mismatch could be identified (Supplemental Fig. 2).

\section{Temporal Pattern and Specificity of RP782 Uptake in Aorta}

To investigate the temporal pattern of MMP activation in the aorta, additional groups of mice underwent RP782 micro-SPECT/CT at 1 and 2 mo after the initiation of the HFD. RP782 uptake along the aorta gradually increased over time, with the mean uptake increasing from $0.13 \pm$ $0.02 \mathrm{cpv} / \mathrm{MBq}$ injected after $1 \mathrm{mo}(n=8)$ to $0.21 \pm 0.03$

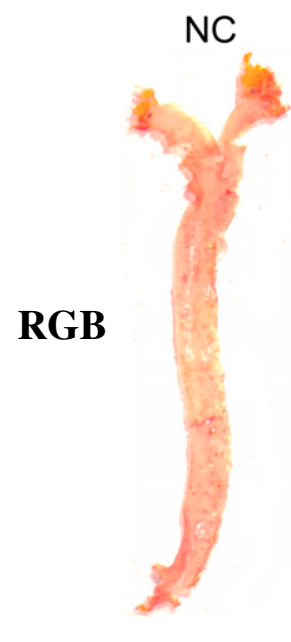

cpv/MBq after $2 \mathrm{mo}(n=8)$ and $0.36 \pm 0.05 \mathrm{cpv} / \mathrm{MBq}$ $(n=4)$ after 3 mo of $\operatorname{HFD}(P<0.01$ between 1 and 3 mo; Fig. 4; Supplemental Fig. 3). RP782 uptake heterogeneity along the aorta appeared more pronounced with progression of atherosclerosis over time. Tracer uptake specificity was demonstrated in a group of animals injected with 50-fold excess of nonlabeled precursor before RP782 administration.
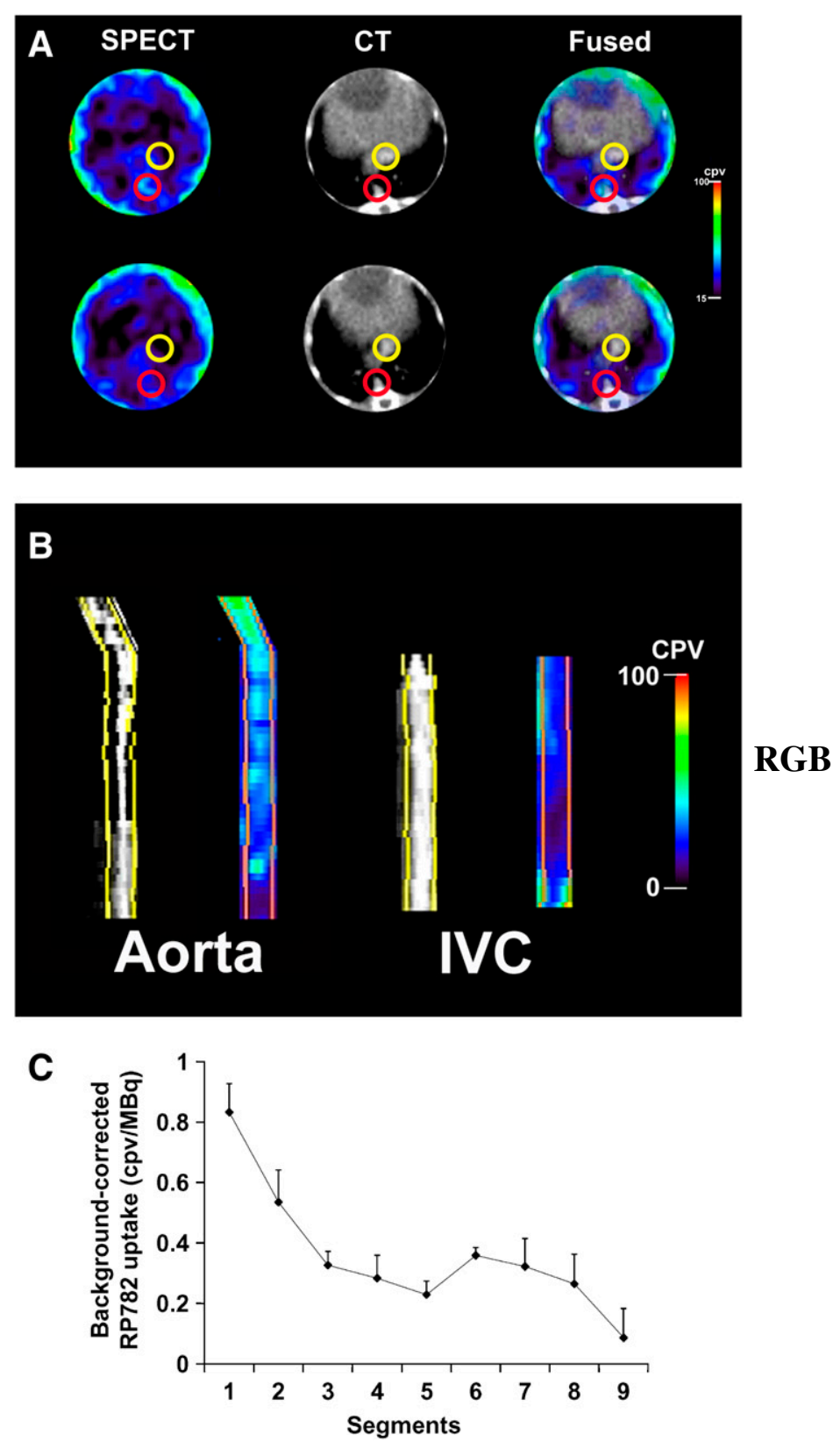

FIGURE 2. RP782 micro-SPECT/CT of MMP activation in atherosclerosis in apoE ${ }^{-1-}$ mice fed HFD for 3 mo. (A) Transversal SPECT, CT, and fused SPECT and CT images demonstrating areas of high (top) and low (bottom) tracer uptake in aorta (red circles). Little tracer uptake can be detected in IVC (yellow circles). (B) Multiplanar reconstruction of SPECT (right) and CT (left) images of aorta and IVC demonstrating heterogeneous RP782 uptake along aorta. (C) Micro-SPECT-derived quantification of RP782 uptake on serial 2$\mathrm{mm}$ segments of aorta, starting at level of aortic arch. $n=4$. 


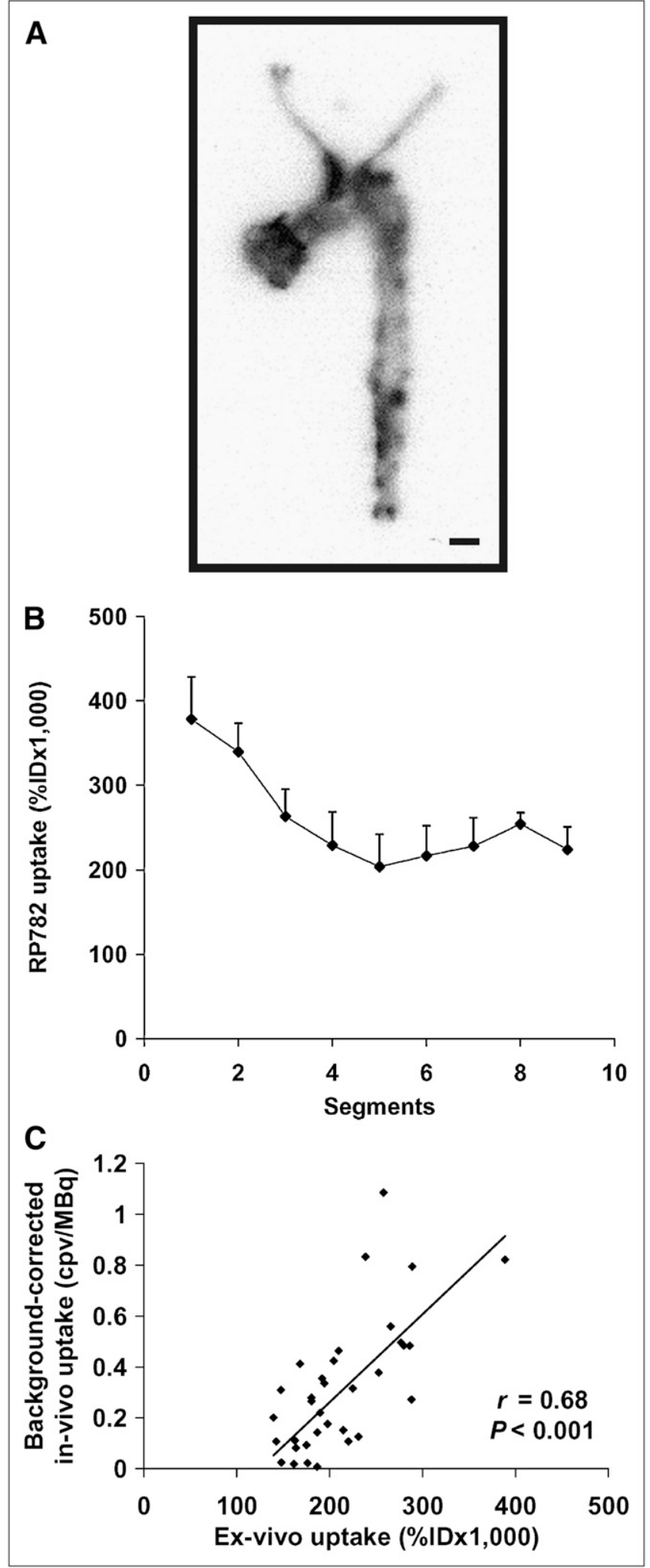

FIGURE 3. (A) Example of RP782 autoradiography demonstrating heterogeneity of tracer uptake in atherosclerotic aorta of mice on HFD for 3 mo. (B) Autoradiography-derived quantification of RP782 uptake on serial 2-mm segments of aorta $(n=4)$. (C) Correlation between micro-SPECT- and autoradiography-derived quantification of RP782 uptake on 2-mm aortic segments. ID = injected dose.

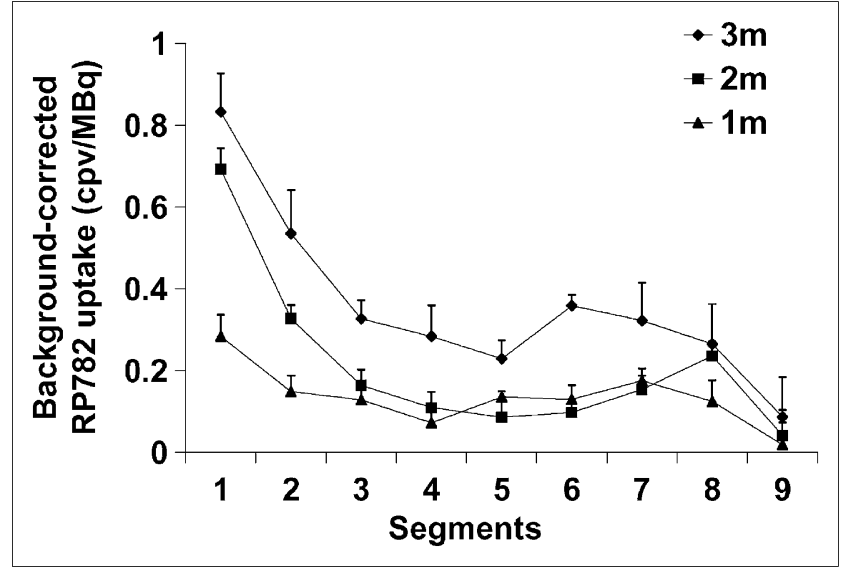

FIGURE 4. Temporal pattern of RP782 uptake in atherosclerotic aorta. Micro-SPECT-derived quantification of RP782 uptake on serial 2-mm segments of aorta of mice fed HFD for $1(n=8), 2$ $(n=8)$, or $3(n=4)$ mo, demonstrating gradual increase in tracer uptake over time.

This led to a significant reduction in RP782 uptake in the aorta (mean aortic arch uptake, $0.24 \pm 0.03 \mathrm{cpv} / \mathrm{MBq}$ injected without precursor, $n=8$, vs. $0.06 \pm 0.04 \mathrm{cpv} / \mathrm{MBq}$ with excess nonlabeled precursor, $n=2, P=0.02$; Fig. 5; Supplemental Fig. 4).

\section{Effect of Dietary Intervention on MMP Activation and Cellular Composition of Atherosclerotic Aorta}

Therapeutic interventions aimed at reducing vascular risk may be associated with a reduction in MMP activity in the vessel wall. To address the effect of dietary intervention on MMP activation in atherosclerosis, animals were placed on normal chow after 2 mo of HFD. Withdrawal from the HFD for 1 mo led to an approximately $30 \%$ reduction in the relative plaque area detected by oil red $\mathrm{O}$ staining, compared with animals kept on HFD for 3 mo $(22.5 \% \pm 0.7 \%$ in the withdrawal group vs. $32.8 \% \pm 4.0 \%$ in the HFD group, $n=3$ in each group, $P=0.01$ ) (Figs. 6A and 6B). A significant (and considerably more pronounced) reduction in aortic MMP activation was detected by RP782 imaging in the withdrawal group (mean aortic uptake, $0.14 \pm 0.05 \mathrm{cpv} / \mathrm{MBq}$ injected in the withdrawal group vs. $0.36 \pm 0.05 \mathrm{cpv} / \mathrm{MBq}$ in the HFD group, $n=4$, $P=0.02$; Figs. 6C and 6D; Supplemental Fig. 5). However, dietary intervention did not appear to alter the heterogeneity of MMP activation along the aorta. Importantly, whereas the withdrawal of the HFD had no effect on aortic CD31 (endothelial cell marker) and SM $\alpha$-actin (vascular SM cell marker) messenger RNA expression (Supplemental Fig. 6), it significantly reduced aortic CD68 and EMR-1 expression, reflecting a reduction in vessel wall macrophage infiltration after dietary intervention $(P<0.05$, Fig. 7).

\section{Biologic Correlates of Heterogeneity of RP782 Uptake in Atherosclerotic Aorta}

To identify the biologic correlates of RP782 uptake, MMP expression and cellular composition of the vessel wall were 


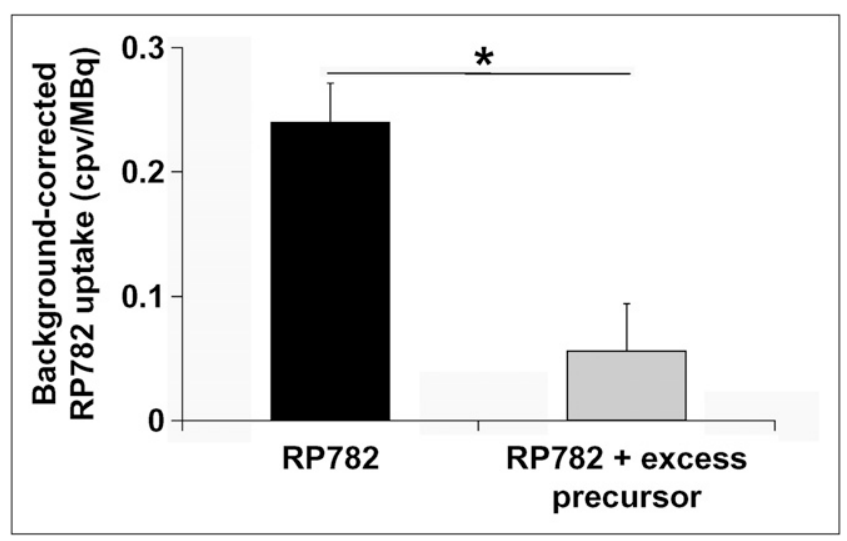

FIGURE 5. RP782 uptake specificity in atherosclerosis. MicroSPECT-derived quantification of RP782 signal in aortic arch in animals without or with injection with 50 -fold excess of unlabeled precursor before tracer administration. ${ }^{\star} P=0.02$.

assessed in 4 segments at regular intervals along the aorta. Similar to imaging data, there was considerable regional variation in the expression of most members of the MMP family studied (with the notable exception of MMP-9), which paralleled and significantly correlated with MMP activation in the same segments detected by micro-SPECT (Supplemental Fig. 7; Table 1). Dietary intervention led to a significant reduction in MMP-2, MMP-3, MMP-12, and MMP-13 (but not MMP-9) messenger RNA expression in the proximal aorta (Supplemental Fig. 7). Several vascular cells, including endothelial cells, vascular SM cells, and monocyte-macrophages, can produce MMPs and contribute to MMP proteolytic activity in the aorta. Although we found no significant correlation between CD31 (endothelial cells) or SM $\alpha$-actin (vascular SM cells) expression and RP782 uptake, CD68 expression (reflecting the presence of macrophages) significantly correlated with MMP activation detected by in vivo imaging $(r=0.65, P=0.04$, Table 1$)$.

\section{DISCUSSION}

Molecular imaging is emerging as a potentially powerful tool for in vivo quantitative assessment of vessel wall biology in atherosclerosis $(3,4)$. Despite differences from human pathology, murine models are important experimental tools for preclinical studies of atherosclerosis. However, the small size of the mouse raises several technical challenges that hamper access to the full potential of molecular imaging in this species. To circumvent this problem, radionuclide-based molecular imaging studies of atherosclerosis in murine models have relied on ex vivo measurements to obtain quantitative data on plaque biology, including local MMP activity (5,7,14-16). Here, we expand the scope of previous studies to image and quantify in vivo MMP activation in murine atherosclerosis by microSPECT/CT. Considerable heterogeneity of MMP activation along atherosclerotic mouse aorta was demonstrated by in vivo imaging, correlating well with the presence of macrophages. Investigating the effect of dietary intervention on MMP activation and its heterogeneity in atherosclerosis, we detected changes in vessel wall biology that extend beyond the expected effect on plaque development.

MMPs are a large family of specialized endopeptidases specific for extracellular matrix and other proteins (17). Their enzymatic activity is dependent on the expression

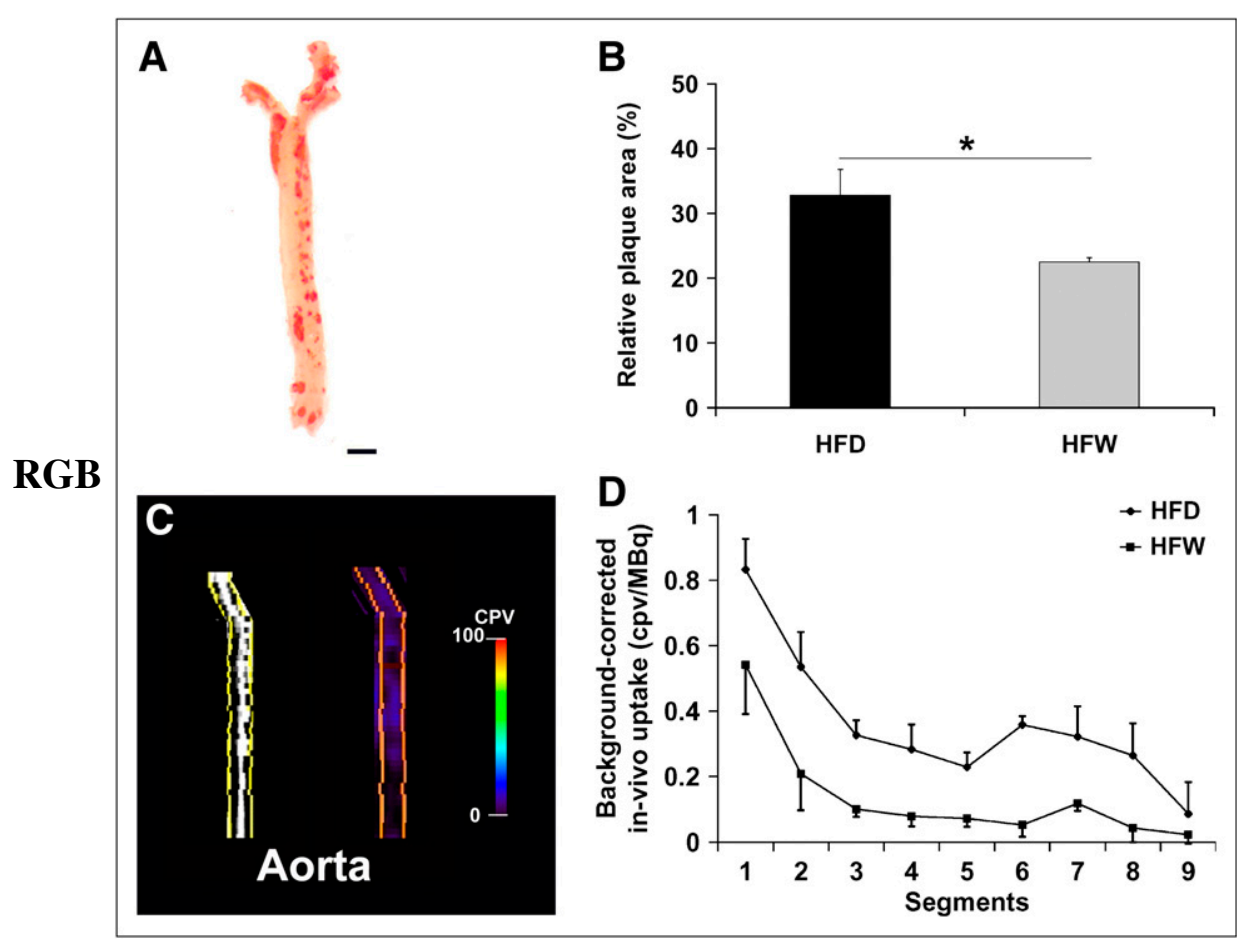

FIGURE 6. (A) Representative example of oil red $\mathrm{O}$ staining of aorta of apoE $\mathrm{E}^{-/-}$mouse fed HFD for $2 \mathrm{mo}$, followed by normal chow for $1 \mathrm{mo}$. Scale bar $=2 \mathrm{~mm}$. (B) Relative plaque area detected by oil red $\mathrm{O}$ staining of aortae of animals fed HFD for 3 mo or HFD for 2 mo, followed by 1 mo of normal chow (HFW) ( $n=3$ in each group). ${ }^{*} P=$ 0.01. (C) Representative examples of multiplanar reconstruction of SPECT (left) and CT (right) images of aorta in HFW group. (D) Micro-SPECT-derived quantification of RP782 uptake in HFD and HFW animals ( $n=4$ in each group). 
FIGURE 7. GAPDH-normalized CD68 and EMR-1 messenger RNA expression detected by real-time RT-PCR in aortae of animals fed HFD for 3 mo or HFD for 2 mo, followed by 1 mo of normal chow (HFW) ( $n=3$ in each group). ${ }^{\star} P<0.05$.

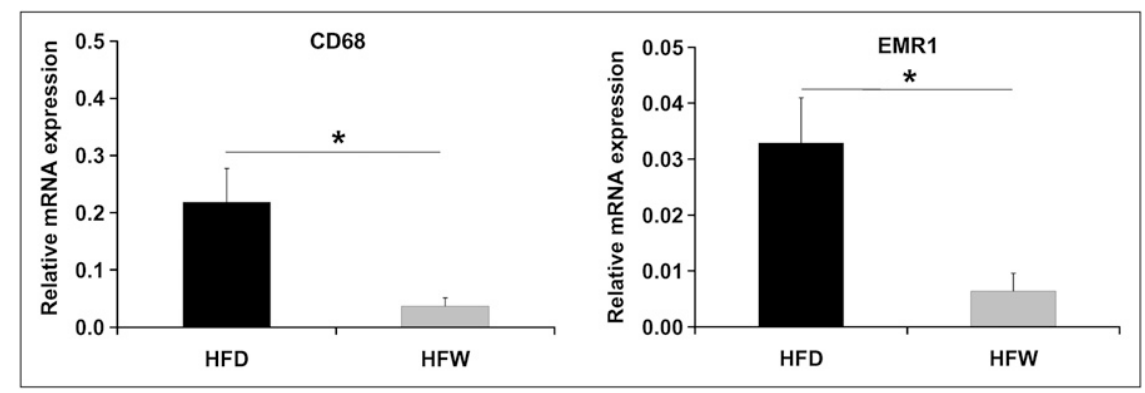

level, activation state, and presence of inhibitors. MMPs play a key role in the development of atherosclerosis and its complications through remodeling of extracellular matrix and activation of cytokines, chemokines, and growth factors. ECs, vascular SM cells, and inflammatory cells, including monocytes and macrophages, are the main sources of MMP activity in the vessel wall (17). The critical role of MMPs in the pathogenesis of atherosclerosis is supported by several lines of evidence, including observations in MMP knockout and transgenic mice (17). For example, atherogenesis is reduced in MMP-2-deficient $a p o E^{-1-}$ mice (18) whereas deletion of MMP-9 appears to have an opposite effect, promoting plaque development (19). In addition to their role in the development of atherosclerosis, MMPs modulate plaque stability through regulation of extracellular matrix protein deposition and degradation, vascular SM cell proliferation, and inflammation (17,20,21). In apoE/MMP-12 double-knockout mice, compared with controls, brachiocephalic artery atherosclerotic lesions show increased vascular SM cells and reduced macrophage content, features associated with plaque stability (19). Similarly, vascular SM cell content is increased in apoE/MMP-7 double-knockout mice, whereas knocking down MMP-3 or MMP-9 confers features of plaque instability (19).

MMP-targeted imaging appears as a promising approach to atherosclerotic plaque characterization for identifying high-risk features. Several studies have demonstrated the feasibility of imaging MMPs (and other relevant targets) using various imaging modalities in animal models of atherosclerosis $(5,8,15,22,23)$. The high sensitivity of nuclear imaging (PET, SPECT) and its applicability to human studies make it an especially promising approach for studies of vessel wall biology in vivo. To acquire quantitative information on MMP activation pattern in atherosclerosis, we used RP782, an ${ }^{111}$ In-labeled tracer with specificity for MMP activation epitope (11), to image MMP activation along the aorta in atherosclerotic apoE ${ }^{-/-}$mice. The use of ${ }^{111}$ In-labeled RP782, rather than a ${ }^{99 m}$ Tc-labeled homo$\log$, allowed us to analyze the samples by relatively highquality autoradiography. Micro-SPECT was combined with CT angiography to localize major blood vessels with accuracy. Despite potential confounding factors, including partial-volume effect and scatter from adjacent structures, we found a strong correlation between in vivo and ex vivo measurements of RP782 uptake along the aorta, validating our approach to in vivo quantification. Comparison of ex vivo and in vivo quantification indicates overcorrection of the background activity on in vivo images, possibly explained by our choice of the area anterior to thoracic aorta, which probably has some degree of specific tracer uptake, to calculate the background activity.

In vivo images showed considerable heterogeneity of MMP activation along the aorta, as was confirmed by autoradiography. In general, aortic segments with prominent atherosclerotic lesions were found to have high levels of RP782 uptake. However, there were areas with discordance between the presence of atherosclerotic plaque and MMP tracer uptake. A similar heterogeneity of plaque distribution, biology, and composition has been observed in animal $(7,24)$ and human $(25-27)$ studies of atherosclerosis. In rabbit atherosclerosis, plaque temperature heterogeneity detected by thermography has been linked to macrophage content and MMP-9 activity (24). In our study, in addition to heterogeneity of MMP expression and activation along the aorta, we detected considerable heterogeneity of MMP activity and expression in adjacent $100-\mu \mathrm{m}$ segments of atherosclerotic aorta with comparable histology (data not shown). This heterogeneity of MMP expression is also present in human carotid atherectomy samples and has been attributed to lesion location, size, and composition (28). The potential sampling errors caused by this remarkable heterogeneity of MMP expression in atherosclerosis high-

TABLE 1

Correlation Between RP782 Uptake In Vivo and Messenger RNA Expression Level in Serial 4-mm Segments of Aorta

\begin{tabular}{llc}
\hline Marker & $r$ & $P$ \\
\hline MMP-2 & 0.7 & 0.03 \\
MMP-3 & 0.81 & 0.008 \\
MMP-9 & 0.18 & NS \\
MMP-12 & 0.66 & 0.04 \\
MMP-13 & 0.9 & 0.003 \\
CD68 & 0.65 & 0.04 \\
$\alpha$-actin & 0.58 & NS
\end{tabular}

Animals were fed HFD for 3 mo or HFD for 2 mo, followed by 1 mo of normal chow.

NS = not significant. 
lights the importance of the more global in vivo assessment of MMP activity that can be achieved by molecular imaging (29). Interestingly, withdrawal of the HFD in our study led to a significant reduction in MMP activation in the aorta. However, the heterogeneity of MMP activation along the aorta persisted in the withdrawal group. The relevance of this observation to human coronary artery disease and its potential clinical implications remain to be determined.

Plaque heterogeneity also indicates that immunostaining of random histologic sections is not a reliable approach to quantification of protein expression or cell infiltration in atherosclerosis. Therefore, to identify correlates of tracer uptake in vivo, we quantified MMP expression and cellular content of aortic segments by real-time RT-PCR. Expression of several MMPs, with the notable exception of MMP-9, correlated well with tracer uptake in atherosclerotic aorta, highlighting the concerted changes of MMP expression in atherosclerosis. The exact role of MMP-9 in atherosclerosis remains controversial (19,30-32), and further studies to address these discrepancies are warranted. Plaque composition, for example, the presence of macrophages, is a key determinant of its propensity to rupture $(1,33)$. Although we did not detect any significant correlation between tracer uptake and CD31 or SM $\alpha$-actin expression, MMP activation detected by molecular imaging correlated well with expression of CD68, a macrophage marker. Withdrawal from HFD led to a significant reduction in aortic macrophage-but not endothelial cells or vascular SM cell-content in parallel with the significant reduction in RP782 uptake. Importantly, the reduction in MMP tracer uptake (as well as macrophage markers) extended beyond the modest reduction in aortic plaque area, indicating the presence of a more stable phenotype in the withdrawal group.

\section{CONCLUSION}

In vivo imaging and quantification of MMP activation in atherosclerosis provides an opportunity to characterize atherosclerotic lesions in vivo and track the effect of therapeutic interventions on plaque biology. It remains to be determined whether differences in MMP tracer uptake can predict differences in the outcome between different patient populations. If validated as such, MMP targeted imaging could have a major impact on stratifying risk and tracking the effect of therapeutic interventions in atherosclerotic diseases.

\section{DISCLOSURE STATEMENT}

The costs of publication of this article were defrayed in part by the payment of page charges. Therefore, and solely to indicate this fact, this article is hereby marked "advertisement" in accordance with 18 USC section 1734.

\section{ACKNOWLEDGMENTS}

This work was supported by National Institutes of Health R01 HL85093, Program Project HL-70295, and a Depart- ment of Veterans Affairs Merit Award. Simon Robinson and Michael Azure are employees of Lantheus Medical Imaging. Albert Sinusas and Mehran Sadeghi receive experimental tracers from Lantheus Medical Imaging. In addition, Albert Sinusas has received research grants from Lantheus Medical Imaging. No other potential conflict of interest relevant to this article was reported.

\section{REFERENCES}

1. Hansson GK. Inflammation, atherosclerosis, and coronary artery disease. $N$ Engl J Med. 2005;352:1685-1695.

2. Virmani R, Burke AP, Farb A, Kolodgie FD. Pathology of the vulnerable plaque. $J$ Am Coll Cardiol. 2006;47(8, suppl):C13-C18.

3. Nahrendorf M, Sosnovik DE, French BA, et al. Multimodality cardiovascular molecular imaging, part II. Circ Cardiovasc Imaging. 2009;2:56-70.

4. Sadeghi MM, Glover DK, Lanza GM, Fayad ZA, Johnson LL. Imaging atherosclerosis and vulnerable plaque. J Nucl Med. 2010;51(suppl 1):51S-65S.

5. Fujimoto S, Hartung D, Ohshima S, et al. Molecular imaging of matrix metalloproteinase in atherosclerotic lesions: resolution with dietary modification and statin therapy. J Am Coll Cardiol. 2008;52:1847-1857.

6. Winter PM, Morawski AM, Caruthers SD, et al. Molecular imaging of angiogenesis in early-stage atherosclerosis with alpha(v)beta3-integrin-targeted nanoparticles. Circulation. 2003;108:2270-2274.

7. Ishino S, Kuge Y, Takai N, et al. ${ }^{99 \mathrm{~m}} \mathrm{Tc}-$ Annexin A5 for noninvasive characterization of atherosclerotic lesions: imaging and histological studies in myocardial infarction-prone Watanabe heritable hyperlipidemic rabbits. Eur J Nucl Med Mol Imaging. 2007;34:889-899.

8. Lancelot E, Amirbekian V, Brigger I, et al. Evaluation of matrix metalloproteinases in atherosclerosis using a novel noninvasive imaging approach. Arterioscler Thromb Vasc Biol. 2008;28:425-432.

9. Kaufmann BA, Carr CL, Belcik JT, et al. Molecular imaging of the initial inflammatory response in atherosclerosis: implications for early detection of disease. Arterioscler Thromb Vasc Biol. 2010;30:54-59.

10. Nahrendorf M, Waterman P, Thurber G, et al. Hybrid in vivo FMT-CT imaging of protease activity in atherosclerosis with customized nanosensors. Arterioscler Thromb Vasc Biol. 2009;29:1444-1451.

11. Su H, Spinale FG, Dobrucki LW, et al. Noninvasive targeted imaging of matrix metalloproteinase activation in a murine model of postinfarction remodeling. Circulation. 2005;112:3157-3167.

12. Zhang J, Nie L, Razavian M, et al. Molecular imaging of activated matrix metalloproteinases in vascular remodeling. Circulation. 2008;118:1953-1960.

13. Sadeghi MM, Krassilnikova S, Zhang J, et al. Detection of injury-induced vascular remodeling by targeting activated alphavbeta 3 integrin in vivo. Circulation. 2004;110:84-90.

14. Hartung D, Petrov A, Haider N, et al. Radiolabeled monocyte chemotactic protein 1 for the detection of inflammation in experimental atherosclerosis. $\mathrm{J} \mathrm{Nucl}$ Med. 2007;48:1816-1821.

15. Ohshima S, Petrov A, Fujimoto S, et al. Molecular imaging of matrix metalloproteinase expression in atherosclerotic plaques of mice deficient in apolipoprotein e or low-density-lipoprotein receptor. J Nucl Med. 2009;50: 612-617.

16. Laitinen I, Marjamaki P, Nagren K, et al. Uptake of inflammatory cell marker $\left[{ }^{11} \mathrm{C}\right] \mathrm{PK} 11195$ into mouse atherosclerotic plaques. Eur J Nucl Med Mol Imaging. 2009;36:73-80.

17. Galis ZS, Khatri JJ. Matrix metalloproteinases in vascular remodeling and atherogenesis: the good, the bad, and the ugly. Circ Res. 2002;90: 251-262.

18. Kuzuya M, Nakamura K, Sasaki T, Cheng XW, Itohara S, Iguchi A. Effect of MMP-2 deficiency on atherosclerotic lesion formation in apoE-deficient mice. Arterioscler Thromb Vasc Biol. 2006;26:1120-1125.

19. Johnson JL, George SJ, Newby AC, Jackson CL. Divergent effects of matrix metalloproteinases $3,7,9$, and 12 on atherosclerotic plaque stability in mouse brachiocephalic arteries. Proc Natl Acad Sci USA. 2005;102:15575-15580.

20. Dollery CM, Libby P. Atherosclerosis and proteinase activation. Cardiovasc Res. 2006;69:625-635.

21. Newby AC. Dual role of matrix metalloproteinases (matrixins) in intimal thickening and atherosclerotic plaque rupture. Physiol Rev. 2005;85:1-31.

22. Amirbekian V, Aguinaldo JG, Amirbekian S, et al. Atherosclerosis and matrix metalloproteinases: experimental molecular MR imaging in vivo. Radiology. 2009;251:429-438. 
23. Ohshima S, Fujimoto S, Petrov A, et al. Effect of an antimicrobial agent on atherosclerotic plaques: assessment of metalloproteinase activity by molecular imaging. J Am Coll Cardiol. 2010;55:1240-1249.

24. Krams R, Verheye S, van Damme LC, et al. In vivo temperature heterogeneity is associated with plaque regions of increased MMP-9 activity. Eur Heart J. 2005;26:2200-2205.

25. Verheye S, De Meyer GR, Van Langenhove G, Knaapen MW, Kockx MM. In vivo temperature heterogeneity of atherosclerotic plaques is determined by plaque composition. Circulation. 2002;105:1596-1601.

26. Stefanadis C, Diamantopoulos L, Vlachopoulos C, et al. Thermal heterogeneity within human atherosclerotic coronary arteries detected in vivo: a new method of detection by application of a special thermography catheter. Circulation. 1999;99:1965-1971.

27. Naghavi M, John R, Naguib S, et al. pH Heterogeneity of human and rabbit atherosclerotic plaques; a new insight into detection of vulnerable plaque. Atherosclerosis. 2002;164:27-35.
28. Choudhary S, Higgins CL, Chen IY, et al. Quantitation and localization of matrix metalloproteinases and their inhibitors in human carotid endarterectomy tissues. Arterioscler Thromb Vasc Biol. 2006;26:2351-2358.

29. Libby P. Perplexity of plaque proteinases. Arterioscler Thromb Vasc Biol. 2006;26:2181-2182.

30. Cho A, Reidy MA. Matrix metalloproteinase-9 is necessary for the regulation of smooth muscle cell replication and migration after arterial injury. Circ Res. 2002;91:845-851.

31. Johnson C, Galis ZS. Matrix metalloproteinase-2 and -9 differentially regulate smooth muscle cell migration and cell-mediated collagen organization. Arterioscler Thromb Vasc Biol. 2004;24:54-60.

32. Gong Y, Hart E, Shchurin A, Hoover-Plow J. Inflammatory macrophage migration requires MMP-9 activation by plasminogen in mice. $J$ Clin Invest. 2008;118:3012-3024.

33. Libby P. Inflammation in atherosclerosis. Nature. 2002;420:868-874. 